\title{
ASYMPTOTIC BOUNDS FOR SPECTRAL BANDS OF PERIODIC SCHRÖDINGER OPERATORS
}

\author{
M. M. SKRIGANOV AND A. V. SOBOLEV \\ Dedicated to L. D. Faddeev on his 70th birthday
}

\begin{abstract}
The precise upper and lower bounds for the multiplicity of the spectrum band overlapping are given for the multidimensional periodic Schrödinger operators with rational period lattices. These bounds are based on very recent results on the lattice point problem.
\end{abstract}

\section{§1. MAIN RESUlT}

1.1. Introduction. The spectral analysis of the Schrödinger operator

$$
H=-\Delta+V
$$

with a real-valued periodic potential is based on the standard Floquet decomposition; see, e.g., [8] or [9]. Let $\Gamma \subset \mathbb{R}^{d}, d \geq 2$, be the period lattice of the potential $V, V(\mathbf{x}+\gamma)=V(\mathbf{x})$ for all $\gamma \in \Gamma$. We denote by $\mathcal{O}$ the standard fundamental set of $\Gamma$, i.e., the parallelepiped (with opposite faces identified) that is spanned by $d$ basis vectors of $\Gamma$ chosen arbitrarily. In other words, $\mathcal{O}$ is the torus $\mathbb{R}^{d} / \Gamma$. The volume of $\mathcal{O}$ is called the determinant of the lattice and is denoted by $\mathrm{d}(\Gamma)$. The symbols $\Gamma^{\dagger}$ and $\mathcal{O}^{\dagger}$ are reserved for the dual lattice and its fundamental set, respectively. If necessary, the dependence on the lattice is reflected in the notation: $\mathcal{O}_{\Gamma}, \mathcal{O}_{\Gamma}^{\dagger}$.

In $\mathfrak{H}=\mathrm{L}^{2}(\mathcal{O})$, we introduce the operators

$$
\begin{aligned}
H_{0}(\mathbf{k}) & =(\mathbf{D}+\mathbf{k})^{2}, \quad \mathbf{D}=-i \nabla, \\
H(\mathbf{k}) & =H_{0}(\mathbf{k})+V
\end{aligned}
$$

depending on the quasimomentum $\mathbf{k} \in \mathcal{O}^{\dagger}$, with the natural domain $\mathrm{H}^{2}(\mathcal{O})$. The spectrum of each $H(\mathbf{k})$ consists of discrete eigenvalues $\lambda_{j}(\mathbf{k})=\lambda_{j}(H(\mathbf{k})), j=1,2, \ldots$, which we arrange in ascending order, counting multiplicity. It is clear that the $\lambda_{j}(\cdot)$ are continuous functions of $\mathbf{k}$. In general, for any bounded selfadjoint continuous operator-valued function $B(\mathbf{k})$, the spectrum of $\hat{H}(\mathbf{k})=H_{0}(\mathbf{k})+B(\mathbf{k})$ is discrete, and the eigenvalues $\lambda_{j}(\hat{H}(\mathbf{k}))$ are continuous functions of $\mathbf{k} \in \mathcal{O}^{\dagger}$. We introduce the counting functions

$$
\begin{aligned}
n(\lambda ; \hat{H}(\mathbf{k})) & =n_{\Gamma}(\lambda ; \hat{H}(\mathbf{k}))=\sharp\left\{j: \lambda_{j}(\hat{H}(\mathbf{k}))<\lambda\right\}, \\
N(\lambda ; \hat{H}(\mathbf{k})) & =N_{\Gamma}(\lambda ; \hat{H}(\mathbf{k}))=\sharp\left\{j: \lambda_{j}(\hat{H}(\mathbf{k})) \leq \lambda\right\}, \quad \mathbf{k} \in \mathcal{O}^{\dagger} .
\end{aligned}
$$

It is clear that

$$
N(\lambda ; \hat{H}(\mathbf{k}))=\lim _{\epsilon \downarrow 0} n(\lambda+\epsilon ; \hat{H}(\mathbf{k}))
$$

2000 Mathematics Subject Classification. Primary 35P15, $11 \mathrm{H} 06$.

Key words and phrases. Periodic operators, lattices.

The first author was supported by RFBR (grant no. 02-01-00086) and by INTAS (grant no. 00-429). 
When $B(\mathbf{k})=V$, we usually write $n(\lambda ; \mathbf{k}), N(\lambda ; \mathbf{k})$. If $V=0$, we write $n_{0}$ and $N_{0}$ instead of $n$ and $N$. The images $\ell_{j}=\bigcup_{\mathbf{k} \in \mathcal{O}^{\dagger}} \lambda_{j}(\mathbf{k})$ of the functions $\lambda_{j}$ are called spectral bands. The spectrum of the initial operator $H$ is the union of the bands: $\sigma(H)=\bigcup_{j} \ell_{j}$. In order to characterize the overlapping of different bands, we introduce the overlap multiplicity:

$$
\mathfrak{m}(\lambda)=\mathfrak{m}_{\Gamma}(\lambda)=\sharp\left\{j \in \mathbb{N}: \lambda \in \ell_{j}\right\}, \quad \lambda \in \mathbb{R},
$$

which shows to how many bands a given value $\lambda$ belongs. The function $\mathfrak{m}(\lambda)$ can be found from the counting functions $n, N$. Let $\mathfrak{n}(\lambda ; \mathbf{k})$ be either of the two functions $N(\lambda ; \mathbf{k})$ or $n(\lambda ; \mathbf{k})$. Denote

$$
\mathfrak{n}^{+}(\lambda)=\max _{\mathbf{k}} \mathfrak{n}(\lambda ; \mathbf{k}), \quad \mathfrak{n}^{-}(\lambda)=\min _{\mathbf{k}} \mathfrak{n}(\lambda ; \mathbf{k}) .
$$

Then the elementary relation

$$
\mathfrak{m}(\lambda)=N^{+}(\lambda)-n^{-}(\lambda)
$$

is proved easily (see, e.g., 9, 10). A $\Gamma$-periodic potential is also $\Lambda$-periodic for any sublattice $\Lambda \subset \Gamma$. Let us find out how the multiplicities $\mathfrak{m}_{\Gamma}(\lambda)$ and $\mathfrak{m}_{\Lambda}(\lambda)$ are related. A simple calculation shows that

$$
\mathfrak{n}_{\Lambda}(\lambda ; \mathbf{k})=\sum_{\boldsymbol{\nu} \in \Lambda^{\dagger} / \Gamma^{\dagger}} \mathfrak{n}_{\Gamma}(\lambda ; \boldsymbol{\nu}+\mathbf{k}), \quad \mathbf{k} \in \mathcal{O}_{\Lambda}^{\dagger} .
$$

This implies the inequalities

$$
\mathfrak{n}_{\Lambda}^{+}(\lambda) \leq \frac{\mathrm{d}(\Lambda)}{\mathrm{d}(\Gamma)} \mathfrak{n}_{\Gamma}^{+}(\lambda), \quad \mathfrak{n}_{\Lambda}^{-}(\lambda) \geq \frac{\mathrm{d}(\Lambda)}{\mathrm{d}(\Gamma)} \mathfrak{n}_{\Gamma}^{-}(\lambda),
$$

whence, by (1.3),

$$
\mathfrak{m}_{\Lambda}(\lambda) \leq \frac{\mathrm{d}(\Lambda)}{\mathrm{d}(\Gamma)} \mathfrak{m}_{\Gamma}(\lambda) .
$$

Our study of $\mathfrak{m}(\lambda)$ is based on the relationship with the counting function for the lattice $\Gamma^{\dagger}$.

1.2. The overlap multiplicity and lattice points counting. For any lattice $\mathrm{M} \subset$ $\mathbb{R}^{d}, d \geq 2$, we denote by $\mathcal{N}[\mathcal{C}]=\mathcal{N}_{\mathrm{M}}[\mathcal{C}]$ the number of lattice points that belong to a bounded set $\mathcal{C} \subset \mathbb{R}^{d}$, i.e.,

$$
\mathcal{N}[\mathcal{C}]=\#\{\gamma \in \mathrm{M}: \boldsymbol{\gamma} \in \mathcal{C}\} .
$$

We are interested in counting the lattice points inside the "shifted" sets $\mathcal{C}$, i.e., inside

$$
\mathcal{C}(\mathbf{k})=\left\{\boldsymbol{\xi} \in \mathbb{R}^{d}: \boldsymbol{\xi}+\mathbf{k} \in \mathcal{C}\right\}, \quad \mathbf{k} \in \mathbb{R}^{d} .
$$

More precisely, for us it is important to have information on the counting function $\mathcal{N}[\cdot]$ for open balls

$$
B(r ; \mathbf{k})=\left\{\boldsymbol{\xi} \in \mathbb{R}^{d}:|\boldsymbol{\xi}+\mathbf{k}|<r\right\}, \quad r>0 .
$$

The functions $\mathcal{N}[\cdot]$ and $n_{0}(\lambda ; \mathbf{k})$ are related in the following way:

$$
n_{0}\left(\rho^{2} ; \mathbf{k}\right)=\mathcal{N}_{\Gamma^{\dagger}}[B(\rho ; \mathbf{k})], \quad \rho \geq 0 .
$$

For all $r>0$ we define

$$
\mathcal{N}_{\mathrm{M}}^{+}(r)=\max _{\mathbf{k}} \mathcal{N}_{\mathrm{M}}[B(r ; \mathbf{k})], \quad \mathcal{N}_{\mathrm{M}}^{-}(r)=\min _{\mathbf{k}} \mathcal{N}_{\mathrm{M}}[B(r ; \mathbf{k})],
$$

and for any $\lambda>0$ and any $\delta$ with $|\delta|<\lambda$ we put

$$
V(\lambda, \delta)=V_{\mathrm{M}}(\lambda, \delta)=\mathcal{N}_{\mathrm{M}}^{+}(\sqrt{\lambda-\delta})-\mathcal{N}_{\mathrm{M}}^{-}(\sqrt{\lambda+\delta}) .
$$


We call this quantity the $\delta$-variation of the lattice counting function at the point $\lambda$. Our aim is to show that the multiplicity $\mathfrak{m}(\lambda)$ can be estimated from both sides by the $\delta$ variation. For convenience, from now on we assume that the mean value of the potential is zero, that is,

$$
\int_{\mathcal{O}} V(\mathbf{x}) d x=0
$$

For general $V$ the results can be recovered in an obvious way.

Theorem 1.1. Let $d \geq 2$, and let $V$ be a continuous real-valued $\Gamma$-periodic function satisfying (1.9). Then for any $\delta>0$ we have

$$
V_{\Gamma^{\dagger}}(\lambda, \delta)-O\left(\lambda^{\varkappa_{d}}\right) \leq \mathfrak{m}(\lambda) \leq V_{\Gamma^{\dagger}}(\lambda ;-\delta)+O\left(\lambda^{\varkappa_{d}}\right)
$$

for large $\lambda$, where

$$
\varkappa_{l}=\frac{l-3}{2}+\frac{1}{l}, \quad l=1,2, \ldots .
$$

The proof of this theorem is postponed until the next section. We list the known properties of the function $\mathcal{N}[B(\rho ; \mathbf{k})]$, starting with a classical bound proved by E. Landau (see [4]) for arbitrary lattices $\mathrm{M} \subset \mathbb{R}^{d}, d \geq 1$ :

$$
\mathcal{N}_{\mathrm{M}}[B(\rho ; \mathbf{k})]=\frac{\mathrm{w}_{d}}{\mathrm{~d}(\mathrm{M})} \rho^{d}+O\left(\rho^{2 \varkappa_{d+1}}\right)
$$

uniformly in $\mathbf{k} \in \mathbb{R}^{d}$. Here $\mathrm{w}_{d}$ is the volume of the unit ball in $\mathbb{R}^{d}$, and $\varkappa_{d}$ is defined in (1.11). Today more precise results are known (see [1] and the references therein). For instance, if $d \geq 5$, then

$$
\mathcal{N}_{\mathrm{M}}[B(\rho ; \mathbf{k})]=\frac{\mathrm{w}_{d}}{\mathrm{~d}(\mathrm{M})} \rho^{d}+O\left(\rho^{d-2}\right)
$$

uniformly in $\mathbf{k}$. The proof of (1.10) is based on (1.12). Should (1.13) be used instead, a more precise remainder estimate $O\left(\lambda^{(d-3) / 2}\right)$ in (1.10) (for $d \geq 6$ ) would be obtained. However, for our purposes, the present more elementary estimate (1.10) suffices.

In order to obtain yet more precise estimates for the remainder in (1.12), we need to distinguish between rational and irrational lattices.

Definition 1.2. A lattice $\Gamma \subset \mathbb{R}^{d}$ is said to be rational if for any two vectors $\gamma_{1}, \gamma_{2} \in \Gamma$ their inner product satisfies the relation

$$
\left\langle\gamma_{1}, \gamma_{2}\right\rangle=\beta_{\Gamma} r_{12},
$$

where $\beta_{\Gamma} \neq 0$ is a real constant independent of $\gamma_{1}, \gamma_{2}$, and $r_{12}=r_{21}$ is an integer. Otherwise the lattice is irrational.

It is clear that, in order to check the rationality of a lattice $\Gamma$, it suffices to verify (1.14) only for the basis vectors of $\Gamma$. Note also that for any rational (respectively, irrational) lattice $\Gamma$ the dual lattice $\Gamma^{\dagger}$ is also rational (respectively, irrational).

In [1] it was shown that the remainder in (1.13) can be replaced by $o\left(\rho^{d-2}\right)$ if and only if the lattice $\mathrm{M}$ is irrational. For any $\delta>0$, from (1.8) and (1.13) it follows at once that

$$
V_{\mathrm{M}}(\lambda,-\delta) \leq C(\delta) \lambda^{\frac{d-2}{2}}, \quad d \geq 5,
$$

and

$$
\lim _{\delta \rightarrow 0} \limsup _{\lambda \rightarrow \infty} \lambda^{-\frac{d-2}{2}} V_{M}(\lambda,-\delta)=0 \quad \text { if } \mathrm{M} \text { is irrational and } d \geq 5,
$$

for large $\lambda$. For rational lattices the corresponding lower bound was proved in [1]. 
Proposition 1.3 (11]). Let $\mathrm{M}$ be a rational lattice, and let $d \geq 4$. Then there are three positive constants $\delta_{0}=\delta_{0}(\mathrm{M}), \lambda_{0}=\lambda_{0}(\mathrm{M})$, and $c_{\mathrm{M}}$ such that for all $\delta \in\left[0, \delta_{0}\right]$ and all $\lambda \geq \lambda_{0}$ we have

$$
V_{\mathrm{M}}(\lambda, \delta) \geq \begin{cases}c_{\mathrm{M}} \lambda^{\frac{d-2}{2}}, & d \geq 5, \\ c_{\mathrm{M}}(\log \log \lambda)^{-1} \lambda, & d=4, \\ c_{\mathrm{M}} \lambda, & d=4, \mathrm{M}=\mathbb{Z}^{4} .\end{cases}
$$

In combination with Theorem 1.1, the above properties of rational and irrational lattices lead to the following theorem, which constitutes the main result of the paper.

Theorem 1.4. Let $d \geq 4$, and let the lattice $\Gamma$ be rational. Suppose $V$ satisfies the conditions of Theorem 1.1, Then there exist positive constants $\lambda_{0}=\lambda_{0}(\Gamma, V)$ and $c_{\Gamma}, C_{\Gamma}$ such that for all $\lambda \geq \lambda_{0}$ we have

$$
c_{\Gamma} \lambda^{\frac{d-2}{2}} \leq \mathfrak{m}_{\Gamma}(\lambda) \leq C_{\Gamma} \lambda^{\frac{d-2}{2}}
$$

if $d \geq 5$, and

$$
\mathfrak{m}_{\Gamma}(\lambda) \geq c_{\Gamma}(\log \log \lambda)^{-1} \lambda
$$

if $d=4$. If $d=4$ and $\Gamma$ contains a cubic sublattice, then

$$
\mathfrak{m}_{\Gamma}(\lambda) \geq c_{\Gamma} \lambda \text {. }
$$

The constants $C_{\Gamma}, c_{\Gamma}$ depend on the lattice $\Gamma$ and do not depend on $V$.

The corresponding result for irrational lattices is the following

Theorem 1.5. Let $\Gamma \subset \mathbb{R}^{d}, d \geq 5$, be an irrational lattice, and let $V$ satisfy the conditions of Theorem 1.1. Then

$$
\mathfrak{m}_{\Gamma}(\lambda)=o\left(\lambda^{\frac{d-2}{2}}\right), \quad \lambda \rightarrow \infty .
$$

Proof of Theorem 1.4. Let $\delta \in\left(0, \delta_{0}\right]$ be a number such that estimates (1.17) are fulfilled. Then the lower bounds in (1.18), (1.19) follow from Theorem 1.1. since $\varkappa_{d}<(d-2) / 2$. The upper bound in (1.18) follows from Theorem 1.1 and (1.15).

If $d=4$ and $\Gamma$ contains a cubic sublattice $\Lambda$, we view $V$ as being $\Lambda$-periodic. By a straightforward scaling transformation, $\Lambda$ is reduced to $\mathbb{Z}^{4}$, so that the bound (1.17) for $\mathbb{Z}^{4}$, Theorem 1.1] and (1.4) yield (1.20).

Proof of Theorem 1.5. The argument is much more elementary than in the preceding proof. Since $\varkappa_{d}<(d-2) / 2$, Theorem 1.1 shows that, for any $\delta>0$,

$$
\limsup _{\lambda \rightarrow \infty} \lambda^{-\frac{d-2}{2}} \mathfrak{m}_{\Gamma}(\lambda)=\limsup _{\lambda \rightarrow \infty} \lambda^{-\frac{d-2}{2}} V_{\Gamma^{\dagger}}(\lambda,-\delta) .
$$

Since $\delta>0$ is arbitrary, the bound (1.21) immediately follows from (1.16).

Comparing (1.21) with (1.18), we see that the overlap multiplicity is sensitive (at least for $d \geq 5$ ) to the arithmetic properties of the underlying lattice: the lower bound in (1.18) is impossible if the lattice is irrational.

Note that for $d=4$, Theorem 1.1 and estimate (1.12) immediately lead to the upper bound

$$
\mathfrak{m}_{\Gamma}(\lambda) \leq C \lambda^{\frac{6}{5}} .
$$

However, this estimate is of a different order than the lower bound (1.19), and for this reason we did not include it in Theorem 1.4. It should be noted nevertheless that for rational lattices $\mathrm{M}$ in $\mathbb{R}^{4}$ it is known (see [5] and [3, Chapter 4]) that

$$
\mathcal{N}_{\mathrm{M}}[B(\rho ; \mathbf{0})]=\frac{\mathrm{w}_{4}}{\mathrm{~d}(\mathrm{M})} \rho^{4}+O\left(\rho^{2}(\log \rho)^{2}\right) .
$$


The authors do not know whether such a bound is available for arbitrary k. Should it be the case, Theorem 1.1 would provide the upper bound

$$
\mathfrak{m}_{\Gamma}(\lambda) \leq C_{\Gamma} \lambda(\log \lambda)^{2}
$$

for all rational lattices $\Gamma \subset \mathbb{R}^{4}$, which is the same as (1.20), at least in the power scale.

It is instructive to rephrase Theorem 1.4 in the following way.

Corollary 1.6. Let $d \geq 4$, and let $\Gamma$ be a rational lattice. Suppose that $V$ is as in Theorem 1.1. Then

$$
\begin{aligned}
\lim _{\lambda \rightarrow \infty} \frac{\log \mathfrak{m}(\lambda)}{\log \lambda}=\frac{d-2}{2}, & d \geq 5, \\
\liminf _{\lambda \rightarrow \infty} \frac{\log \mathfrak{m}(\lambda)}{\log \lambda} \geq 1, & d=4 .
\end{aligned}
$$

Estimates (1.22) and (1.23) follow from (1.18) and (1.19), respectively. Certainly, rewriting (1.18) -(1.20) by using the log-function leads to a loss of information. On the other hand, formulas (1.22) and (1.23) have a more invariant form than (1.18)-(1.20), because their right-hand sides do not depend on the choice of the lattice $\Gamma$.

Again we emphasize that the upper bounds in Theorem 1.4 follow from the known asymptotic estimate (1.13), but the lower bounds require the new Proposition 1.3. Lower bounds of the form (1.18), (1.19) were proved initially in [9], and there the asymptotics (1.22) was stated as a conjecture (see [9, Introduction, (0.12)]. Although the methods of [9] are also based on the lattice point counting, the introduction of the $\delta$-variation in [1] substantially simplified the proofs of the lower bounds by separating the number-theoretic part of the argument from the spectral one.

Theorem 1.4 implies in particular that the number of gaps in the spectrum of $H$ is finite. This fact, known as the Bethe-Sommerfeld conjecture, was proved initially for rational lattices and $d \geq 4$ in [9], and for arbitrary lattices and $d=3$ in [10. For further discussion and references we refer to [9, 10, 2, 6, 7].

\section{§2. Reduction to A Lattice points problem}

2.1. An intermediate estimate. In this section we reduce the study of the spectrum counting function $n(\lambda ; \mathbf{k})$ to that of the lattice counting function $\mathcal{N}_{\Gamma^{\dagger}}(r ; \mathbf{k})$.

Theorem 2.1. For $d \geq 2$, suppose that $V$ satisfies the conditions of Theorem 1.1, Then for any $\delta>0$ there are two constants $\lambda_{0}=\lambda_{0}(\delta, V)>0$ and $C=C(\delta)$ such that for all $\lambda \geq \lambda_{0}$ the distribution function $n(\lambda ; \mathbf{k})$ satisfies the estimate

$$
\mathcal{N}_{\Gamma^{\dagger}}[B(\sqrt{\lambda-\delta} ; \mathbf{k})]-C \lambda^{\varkappa} \leq n(\lambda ; \mathbf{k}) \leq \mathcal{N}_{\Gamma^{\dagger}}[B(\sqrt{\lambda+\delta} ; \mathbf{k})]+C \lambda^{\varkappa},
$$

where $\varkappa=\varkappa_{d}$ is defined in (1.11).

Before proving Theorem 2.1 we show how to deduce Theorem 1.1 from it.

Proof of Theorem 1.1. We use Theorem 2.1 for some fixed $\delta>0$ and $\delta^{\prime}<\delta$. By the definitions (1.2) and (1.7), we have

$$
\mathcal{N}_{\Gamma^{\dagger}}^{-}(\sqrt{\lambda-\delta})-C \lambda^{\varkappa} \leq n^{-}(\lambda) \leq \mathcal{N}_{\Gamma^{\dagger}}^{-}(\sqrt{\lambda+\delta})+C \lambda^{\varkappa}
$$

and, by (1.1),

$$
\mathcal{N}_{\Gamma^{\dagger}}^{+}(\sqrt{\lambda-\delta})-C \lambda^{\varkappa} \leq N^{+}(\lambda) \leq \mathcal{N}_{\Gamma^{\dagger}}^{+}\left(\sqrt{\lambda+\epsilon+\delta^{\prime}}\right)+C \lambda^{\varkappa}
$$

for any $\epsilon>0$. Take $\epsilon=\delta-\delta^{\prime}$. Now (1.10) follows from the definitions (1.3) and (1.8). 
We note in advance that in the proof of the theorem we only need one piece of information on the lattice counting function: the elementary estimate (1.12).

We begin the proof of Theorem 2.1 with the following useful observation. If $V(\mathbf{x})$ and $\tilde{V}(\mathbf{x})$ are two continuous $\Gamma$-periodic potentials and

$$
\delta^{\prime}=\sup _{\mathbf{x}}|V(\mathbf{x})-\tilde{V}(\mathbf{x})|,
$$

then an elementary perturbation argument leads to the estimate

$$
N\left(\lambda-\delta^{\prime} ; H(\mathbf{k})\right) \leq N(\lambda ; \tilde{H}(\mathbf{k})) \leq N\left(\lambda+\delta^{\prime} ; H(\mathbf{k})\right)
$$

Consequently, if estimate (2.1) is valid with a parameter $\delta=\delta_{0}>0$ for some given potential $V$, then it is also valid for any other potential $\tilde{V}$ with $\delta=\delta_{0}+\delta^{\prime}$. Thus, it suffices to prove Theorem 2.1 for potentials from a set that is dense in the class of all real-valued periodic continuous functions with zero mean. As such a set, it is convenient to take the set of all real trigonometric polynomials with zero mean value; so, we assume that

$$
V(\mathbf{x})=\sum_{\boldsymbol{\theta} \in \Theta} V_{\boldsymbol{\theta}}(\mathbf{x}), \quad V_{\boldsymbol{\theta}}(\mathbf{x})=v_{\boldsymbol{\theta}} e^{i\langle\mathbf{x}, \boldsymbol{\theta}\rangle},
$$

where $v_{\boldsymbol{\theta}}=\overline{v_{-\boldsymbol{\theta}}}$ are the Fourier coefficients of the function $V$ and $\Theta \subset \Gamma^{\dagger}, \Theta \not \supset\{\mathbf{0}\}$, is a finite subset of the dual lattice. Observe that the requirement $V=\bar{V}$ implies that the set $\Theta$ is symmetric in the sense that $\boldsymbol{\theta}$ and $-\boldsymbol{\theta}$ belong or do not belong to $\Theta$ simultaneously.

2.2. An auxiliary geometric construction. Let $M \subset \mathbb{R}^{d}$ be an arbitrary lattice, and let $\boldsymbol{\theta} \in \mathbf{M}, \boldsymbol{\theta} \neq \mathbf{0}$, be a fixed vector. We introduce the following orthogonal coordinates in $\mathbb{R}^{d}$ :

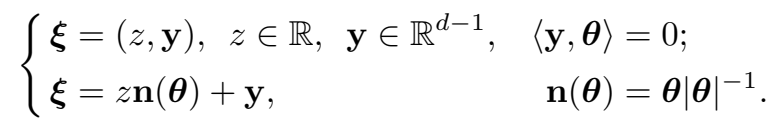

We fix two positive numbers $A$ and $B$ satisfying

$$
A>|\boldsymbol{\theta}|^{2}, \quad 4|\boldsymbol{\theta}|^{-2} A^{2}+A<B,
$$

consider the spherical shell

$$
S(\lambda)=\left\{\boldsymbol{\xi} \in \mathbb{R}^{d}: \lambda-A<|\boldsymbol{\xi}|^{2}<\lambda+A\right\},
$$

and split it into two disjoint components:

$$
\begin{aligned}
S(\lambda) & =\Omega_{\boldsymbol{\theta}}(\lambda) \cup \Lambda_{\boldsymbol{\theta}}(\lambda) \\
\Omega_{\boldsymbol{\theta}}(\lambda) & =\left\{\boldsymbol{\xi} \in S(\lambda):|\mathbf{y}|^{2}>\lambda-B\right\}, \\
\Lambda_{\boldsymbol{\theta}}(\lambda) & =\left\{\boldsymbol{\xi} \in S(\lambda):|\mathbf{y}|^{2} \leq \lambda-B\right\} .
\end{aligned}
$$

In all the lemmas that follow we assume that $\lambda \geq \lambda_{0}$ with a sufficiently large number $\lambda_{0}=\lambda_{0}(A, B)>0$. First, we estimate the number of lattice points in the set $\Omega_{\boldsymbol{\theta}}(\lambda ; \mathbf{k})$ defined as in (1.5).

Lemma 2.2. For any lattice $\mathrm{M} \subset \mathbb{R}^{d}, d \geq 2$, and any numbers $A, B$ satisfying (2.4), the estimate

$$
\max _{\mathbf{k} \in \mathbb{R}^{d}} \mathcal{N}_{\mathrm{M}}\left[\Omega_{\boldsymbol{\theta}}(\lambda ; \mathbf{k})\right] \leq C \lambda^{\varkappa_{d}}
$$

is fulfilled with a constant $C=C(\boldsymbol{\theta}, A, B)$ independent of $\lambda$. 
Proof. Instead of working with $\Omega_{\boldsymbol{\theta}}$, we estimate the number of lattice points in the set $W_{\boldsymbol{\theta}}(\lambda ; \mathbf{k})$ with

$$
W_{\boldsymbol{\theta}}(\lambda)=\left\{\boldsymbol{\xi} \in \mathbb{R}^{d}:|z|^{2} \leq A+B, \lambda-B<|\mathbf{y}|^{2}<\lambda+B\right\},
$$

which contains $\Omega_{\boldsymbol{\theta}}(\lambda ; \mathbf{k})$ because $B>A$. Let $\Pi=\Pi_{\boldsymbol{\theta}}$ be the hyperplane in $\mathbb{R}^{d}$ defined by the condition $z=0$, and let $P$ be the orthogonal projection in $\mathbb{R}^{d}$ onto $\Pi$. By the definition of $W_{\boldsymbol{\theta}}$, we have

$$
P W_{\boldsymbol{\theta}}(\lambda ; \mathbf{k})=\left\{\mathbf{y} \in \Pi: \lambda-B<|\mathbf{y}+P \mathbf{k}|^{2}<\lambda+B\right\} .
$$

Obviously, the projection $\Lambda=P \mathrm{M}$ is a $(d-1)$-dimensional lattice, and the points of $\mathrm{M}$ lie on the straight lines

$$
\ell_{\boldsymbol{\eta}}=\left\{\boldsymbol{\xi} \in \mathbb{R}^{d}: \boldsymbol{\xi}=\boldsymbol{\eta}+t \boldsymbol{\theta}, t \in \mathbb{R}\right\}, \quad \boldsymbol{\eta} \in \Lambda .
$$

If the intersection $\ell_{\boldsymbol{\eta}}(\lambda, \boldsymbol{\theta}, \mathbf{k})=\ell_{\boldsymbol{\eta}} \cap W_{\boldsymbol{\theta}}(\lambda ; \mathbf{k})$ is not empty, then, by (2.9), this intersection is a straight segment of length $2 \sqrt{A+B}$. Consequently, the number of lattice points $\gamma \in \mathrm{M}$ on $\ell_{\boldsymbol{\eta}}(\lambda, \boldsymbol{\theta}, \mathbf{k})$ does not exceed $O(\sqrt{A+B})$. It remains to show that

$$
\sup _{\mathbf{k} \in \mathbb{R}^{d}} \mathcal{N}_{\Lambda}\left[P W_{\boldsymbol{\theta}}(\lambda ; P \mathbf{k})\right] \leq C \lambda^{\varkappa_{d}} .
$$

Formula (1.12) applied to the $(d-1)$-dimensional lattice $\Lambda$ shows that

$$
\begin{aligned}
\mathcal{N}_{\Lambda}\left[P W_{\boldsymbol{\theta}}(\lambda ; P \mathbf{k})\right] & \\
\leq & \mathcal{N}_{\Lambda}[B(\sqrt{\lambda+B} ; P \mathbf{k})]-\mathcal{N}_{\Lambda}[B(\sqrt{\lambda-B} ; P \mathbf{k})]=O\left(B \lambda^{\frac{d-3}{2}}\right)+O\left(\lambda^{\varkappa_{d}}\right) \\
\quad & =O\left(\lambda^{\varkappa_{d}}\right)
\end{aligned}
$$

uniformly in $\mathbf{k} \in \mathbb{R}^{d}$. The proof of (2.10) and of the lemma is complete.

We emphasize that in the proof of the above lemma we have used only the elementary estimate (1.12) and did not appeal to the more precise, but much more difficult result (1.13).

Lemma 2.3. Let $\boldsymbol{\theta} \in \mathrm{M} \subset \mathbb{R}^{d},|\boldsymbol{\theta}|>0$, be a fixed vector, and let $A, B$ satisfy (2.4). If $\boldsymbol{\xi} \in \Lambda_{\boldsymbol{\theta}}(\lambda)$, then $\boldsymbol{\xi} \pm \boldsymbol{\theta} \notin S(\lambda)$.

Proof. Using the coordinates (2.3) associated with the vector $\boldsymbol{\theta}$, for $\boldsymbol{\xi}=(z, \mathbf{y})$ we get $\boldsymbol{\xi}+\boldsymbol{\theta}=(z+|\boldsymbol{\theta}|, \mathbf{y})$. Since $\boldsymbol{\xi} \in \Lambda_{\boldsymbol{\theta}}(\lambda)$, by (2.8) we have

$$
z^{2}=|\boldsymbol{\xi}|^{2}-|\mathbf{y}|^{2} \geq \lambda-A-\lambda+B=B-A .
$$

On the other hand,

$$
|\boldsymbol{\xi}+\boldsymbol{\theta}|^{2}=|z+| \boldsymbol{\theta}||^{2}+|\mathbf{y}|^{2}=z^{2}+|\mathbf{y}|^{2}+2 z|\boldsymbol{\theta}|+|\boldsymbol{\theta}|^{2},
$$

whence

$$
\begin{aligned}
|| \boldsymbol{\xi}+\left.\boldsymbol{\theta}\right|^{2}-\lambda \mid & \\
& \geq 2|z||\boldsymbol{\theta}|-\left.|| \boldsymbol{\xi}\right|^{2}-\left.\lambda|-| \boldsymbol{\theta}\right|^{2} \geq 2 \sqrt{B-A}|\boldsymbol{\theta}|-A-|\boldsymbol{\theta}|^{2} \geq 2|\boldsymbol{\theta}| \sqrt{B-A}-4 A+2 A \\
& \geq 2 A .
\end{aligned}
$$

Here we have used (2.4). By (2.5), this implies that $\boldsymbol{\xi}+\boldsymbol{\theta} \notin S(\lambda)$, as required.

For the vector $\boldsymbol{\xi}-\boldsymbol{\theta}$ the proof is the same with obvious modifications. 


\section{§3. Proof of Theorem 2.1}

Let $\mathcal{P}(\mathbf{k} ; \mathcal{C})$ denote the projection in $\mathfrak{H}=\mathrm{L}^{2}(\mathcal{O})$ onto the subspace spanned by the orthonormal family of exponentials

$$
\mathbf{e}_{\boldsymbol{\gamma}}(\mathbf{x})=\frac{1}{\sqrt{\mathrm{d}(\Gamma)}} e^{i\langle\boldsymbol{\gamma}, \mathbf{x}\rangle}, \quad \gamma \in \Gamma^{\dagger} \cap \mathcal{C}(\mathbf{k}) .
$$

Let $\mathfrak{H}(\mathbf{k} ; \mathcal{C})=\mathcal{P}(\mathbf{k} ; \mathcal{C}) \mathfrak{H}$. Suppose that a bounded selfadjoint perturbation $B(\mathbf{k})$ is such that $\mathfrak{H}(\mathbf{k} ; \mathcal{C})$ is an invariant subspace for the operator $\hat{H}(\mathbf{k})=H_{0}(\mathbf{k})+B(\mathbf{k})$. It is natural to define the "partial" counting functions $n(\lambda ; \hat{H}(\mathbf{k}), \mathcal{C})$ for $\hat{H}(\mathbf{k})$ restricted to the subspace $\mathfrak{H}(\mathbf{k} ; \mathcal{C})$.

We begin the proof with reducing the operator $H(\mathbf{k})=H_{0}(\mathbf{k})+V$ to the operator $H_{1}(\mathbf{k})=H_{0}(\mathbf{k})+\mathcal{P}(\mathbf{k} ; S(\lambda)) V \mathcal{P}(\mathbf{k} ; S(\lambda))$. In the following lemma it suffices to assume that $V$ is a bounded real-valued function.

Lemma 3.1. Let $V$ be a bounded real-valued function, and let the set $S(\lambda)$ be as defined in (2.5). Then for any $\delta>0$ there exist numbers $A_{0}=A_{0}(\delta, V)$ and $\lambda_{0}=\lambda_{0}(\delta)$ such that for all $A \geq A_{0}$ and $\lambda \geq \lambda_{0}$ we have

$$
n\left(\lambda-\delta ; H_{1}(\mathbf{k})\right) \leq n(\lambda ; H(\mathbf{k})) \leq n\left(\lambda+\delta ; H_{1}(\mathbf{k})\right) .
$$

Proof. For brevity, we denote

$$
\begin{gathered}
S=S(\lambda), \quad K=\mathbb{R}^{d} \backslash S, \quad \mathcal{P}=\mathcal{P}(\mathbf{k} ; S), \quad \mathcal{Q}=\mathbf{I}-\mathcal{P}, \\
H=H(\mathbf{k}), \quad H_{1}=H_{1}(\mathbf{k}), \quad H_{0}=H_{0}(\mathbf{k}) .
\end{gathered}
$$

Write $H$ in the form

$$
H=H_{0}+\mathcal{P} V \mathcal{P}+\mathcal{Q} V \mathcal{Q}+\mathcal{Q} V \mathcal{P}+\mathcal{P} V \mathcal{Q}
$$

We estimate the last two terms on the right-hand side for a function $u \in \mathfrak{H}$ :

$$
\begin{gathered}
\mid(\mathcal{Q} V \mathcal{P}+\mathcal{P} V \mathcal{Q}) u, u) \mid \leq 2\|V\|\|\mathcal{Q} u\|\|\mathcal{P} u\| \\
\leq \delta\|\mathcal{P} u\|^{2}+\delta^{-1}\|V\|^{2}\|\mathcal{Q} u\|^{2},
\end{gathered}
$$

with an arbitrary $\delta>0$. Consequently,

$$
\begin{aligned}
& H \leq \mathcal{P}\left(H_{1}+\delta\right) \mathcal{P}+\mathcal{Q}\left(H_{0}+\|V\|+\delta^{-1}\|V\|^{2}\right) \mathcal{Q} \\
& H \geq \mathcal{P}\left(H_{1}-\delta\right) \mathcal{P}+\mathcal{Q}\left(H_{0}-\|V\|-\delta^{-1}\|V\|^{2}\right) \mathcal{Q} .
\end{aligned}
$$

We put

$$
H^{ \pm}=\mathcal{Q}\left(H_{0} \pm\|V\| \pm \delta^{-1}\|V\|^{2}\right) \mathcal{Q}
$$

so that

$$
\left\{\begin{array}{l}
n(\lambda ; H) \leq n\left(\lambda ; H_{1}-\delta, S\right)+n\left(\lambda ; H^{-}, K\right), \\
n(\lambda ; H) \geq n\left(\lambda ; H_{1}+\delta, S\right)+n\left(\lambda ; H^{+}, K\right) .
\end{array}\right.
$$

Obviously,

$$
n\left(\lambda ; H^{ \pm}, K\right)=n\left(\lambda \mp\|V\| \mp \delta^{-1}\|V\|^{2} ; \mathcal{Q} H_{0} \mathcal{Q}, K\right) .
$$

By (2.6), (1.6), and the definition of $\mathcal{P}$, the spectrum of the operator $\mathcal{Q} H_{0} \mathcal{Q}$ on the subspace $\mathfrak{H}(\mathbf{k} ; K)$ lies outside the interval $(\lambda-A, \lambda+A)$. Consequently, by an elementary perturbation-theoretic argument, for a sufficiently large $A_{0}=A_{0}(\delta, V)$ the right-hand side of (3.3) coincides with

$$
n\left(\lambda ; \mathcal{Q} H_{0} \mathcal{Q}, K\right)=n\left(\lambda \pm \delta ; H_{0}, K\right)=n\left(\lambda \pm \delta ; H_{1}, K\right)
$$


for all $A \geq A_{0}$. Therefore, (3.2) can be rewritten in the form

$$
\begin{aligned}
& n(\lambda ; H) \leq n\left(\lambda+\delta ; H_{1}, S\right)+n\left(\lambda+\delta ; H_{0}, K\right)=n\left(\lambda+\delta ; H_{1}\right), \\
& n(\lambda ; H) \geq n\left(\lambda-\delta ; H_{1}, S\right)+n\left(\lambda-\delta ; H_{0}, K\right)=n\left(\lambda-\delta ; H_{1}\right),
\end{aligned}
$$

as required.

Now we return to our usual assumption that $V$ is a trigonometric polynomial (2.2). We denote

$$
\gamma_{+}=\max _{\boldsymbol{\theta} \in \Theta}|\boldsymbol{\theta}|, \quad \gamma_{-}=\min _{\boldsymbol{\theta} \in \Theta}|\boldsymbol{\theta}|
$$

and assume that the positive constants $A, B$ satisfy

$$
A>\gamma_{+}^{2}, \quad 4 \gamma_{-}^{-2} A^{2}+A<B,
$$

so that (2.4) is satisfied for all $\boldsymbol{\theta} \in \Theta$ simultaneously.

The final step in the reduction is made in the following lemma.

Lemma 3.2. Let $V$ be a trigonometric polynomial (2.2). Let $A, B$ be positive constants satisfying (3.5). Then for all sufficiently large $\lambda$ we have

$$
H_{1}(\mathbf{k})=H_{0}(\mathbf{k})+\sum_{\boldsymbol{\theta} \in \Theta} \mathcal{P}\left(\mathbf{k} ; \Omega_{\boldsymbol{\theta}}(\lambda)\right) V_{\boldsymbol{\theta}} \mathcal{P}\left(\mathbf{k} ; \Omega_{\boldsymbol{\theta}}(\lambda)\right) .
$$

Proof. Formula (2.2) implies that

$$
H_{1}(\mathbf{k})=H_{0}(\mathbf{k})+\sum_{\boldsymbol{\theta} \in \Theta} \mathcal{P}(\mathbf{k} ; S(\lambda)) V_{\boldsymbol{\theta}} \mathcal{P}(\mathbf{k} ; S(\lambda)) .
$$

Therefore, by the definitions (2.7) and (2.8), it suffices to check that

$$
\mathcal{P}(\mathbf{k} ; S(\lambda)) V_{\boldsymbol{\theta}} \mathcal{P}\left(\mathbf{k} ; \Lambda_{\boldsymbol{\theta}}(\lambda)\right)=\mathcal{P}\left(\mathbf{k} ; \Lambda_{\boldsymbol{\theta}}(\lambda)\right) V_{\boldsymbol{\theta}} \mathcal{P}(\mathbf{k} ; S(\lambda))=0,
$$

which is the same as checking the relation

$$
\mathcal{P}(\mathbf{k} ; S(\lambda)) V_{ \pm \boldsymbol{\theta}} \mathcal{P}\left(\mathbf{k} ; \Lambda_{\boldsymbol{\theta}}(\lambda)\right)=0 .
$$

We only need to prove that

$$
V_{ \pm \boldsymbol{\theta}} \mathbf{e}_{\boldsymbol{\gamma}} \notin \mathfrak{H}(\mathbf{k} ; S(\lambda))
$$

if $\gamma+\mathbf{k} \in \Lambda_{\boldsymbol{\theta}}(\lambda)$ (see (3.1) for the definition of $\mathbf{e}_{\gamma}$ ). By the definition of $V_{\boldsymbol{\theta}}$ (see (2.2)), we have

$$
V_{ \pm \boldsymbol{\theta}} \mathbf{e}_{\boldsymbol{\gamma}}=v_{ \pm \boldsymbol{\theta}} \mathbf{e}_{\gamma \pm \boldsymbol{\theta}}
$$

Inequalities (3.5) show that the parameters $A$ and $B$ satisfy (2.4) for all $\boldsymbol{\theta} \in \Theta$. Consequently, by Lemma 2.3, the vectors $\gamma+\mathbf{k} \pm \boldsymbol{\theta}$ do not belong to $S(\lambda)$, that is, $\mathbf{e}_{\gamma \pm \boldsymbol{\theta}} \notin$ $S(\lambda ; \mathbf{k})$, whence we see that (3.7) is satisfied.

Proof of Theorem 2.1. Recall that it suffices to prove the theorem for a trigonometric polynomial of the form (2.2). Let $\delta>0$ be fixed, and let $A_{0}=A_{0}(\delta)$ and $\lambda_{0}(\delta)$ be as in Lemma 3.1. Let $A \geq A_{0}, B>0$ be arbitrary numbers satisfying (3.5). Since the set $\Theta$ is finite, Lemma 3.2 shows that the operator $H_{1}(\mathbf{k})$ is a finite rank perturbation of $H_{0}(\mathbf{k})$, and by Lemma 2.2 we have

$$
\operatorname{dim}\left(H_{1}(\mathbf{k})-H_{0}(\mathbf{k})\right) \leq \sum_{\boldsymbol{\theta} \in \Theta} \operatorname{dim} \mathcal{P}\left(\mathbf{k} ; \Omega_{\boldsymbol{\theta}}(\lambda)\right) \leq C \lambda^{\varkappa_{d}}, \quad C=C(\delta) .
$$

Lemma 3.1 allows us to write the estimate

$$
n\left(\lambda-\delta ; H_{0}(\mathbf{k})\right)-C \lambda^{\varkappa_{d}} \leq n(\lambda ; H(\mathbf{k})) \leq n\left(\lambda+\delta ; H_{0}(\mathbf{k})\right)+C \lambda^{\varkappa_{d}},
$$

which follows by an elementary perturbation argument. Now (2.1) is implied by (1.6). 
As was explained earlier, Theorem 2.1 leads to Theorem 1.1, and consequently, to Theorem 1.4.

Acknowledgement. This paper was written during the visit of the first author to the Department of Mathematics, University of Sussex, in Autumn 2003. The visit was supported by the Royal Society.

\section{REFERENCES}

[1] F. Götze, Lattice point problems and values of quadratic forms, Invent. Math. 157 (2004), 195-226. MR2135188

[2] B. Helffer and A. Mohamed, Asymptotic of the density of states for the Schrödinger operator with periodic electric potential, Duke Math. J. 92 (1998), 1-60. MR1609321 (99e:35166)

[3] E. Krätzel, Lattice points, Math. Appl. (East European Ser.), vol. 33, Kluwer Acad. Publ. Group, Dordrecht, 1988. MR0998378 (90e:11144)

[4] E. Landau, Zur analytischen Zahlentheorie der definiten quadratischen Formen. (Über die Gitterpunkte in einem mehrdimensionalen Ellipsoid), Berichte Math.-Natur. Kl. (Berlin) 31 (1915), $458-476$.

[5] — Über Gitterpunkte in mehrdimensionalen Ellipsoiden, Math. Z. 21 (1924), 126-132.

[6] L. Parnovski and A. V. Sobolev, On the Bethe-Sommerfeld conjecture for the polyharmonic operator, Duke Math. J. 107 (2001), 209-238. MR1823047 (2002d:35050)

[7] _ Lattice points, perturbation theory and the periodic polyharmonic operator, Ann. Henri Poincaré 2 (2001), 573-581. MR1846857 (2002j:35235)

[8] M. Reed and B. Simon, Methods of modern mathematical physics. IV. Analysis of operators, Acad. Press, New York-London, 1978. MR0493421 (58:12429c)

[9] M. M. Srkiganov, Geometric and arithmetic methods in the spectral theory of multi-dimensional periodic operators, Trudy Mat. Inst. Steklov. 171 (1985), 171 pp.; English transl., Proc. Steklov Inst. Math. 1987, no. 2 (171), 121 pp. MR0798454 (87h:47110) MR0905202 (88g:47038)

[10] - The spectrum band structure of the three-dimensional Schrödinger operator with periodic potential, Invent. Math. 80 (1985), 107-121. MR0784531 (86i:35107)

[11] M. M. Skriganov and A. V. Sobolev, Variation of the number of lattice points in large balls, Acta Arith. (2005) (to appear).

St. Petersburg Branch, Steklov Mathematical Institute, Russian Academy of Sciences, Fontanka 27, St. Petersburg 191023, Russia

E-mail address: skrig@pdmi.ras.ru

School of Mathematics, University of Birmingham, Edgbaston Birmingham, B152Tt, United KINGDOM

E-mail address: asobolev@bham.ac.uk

Received 8/APR/2005

Translated by THE AUTHORS 